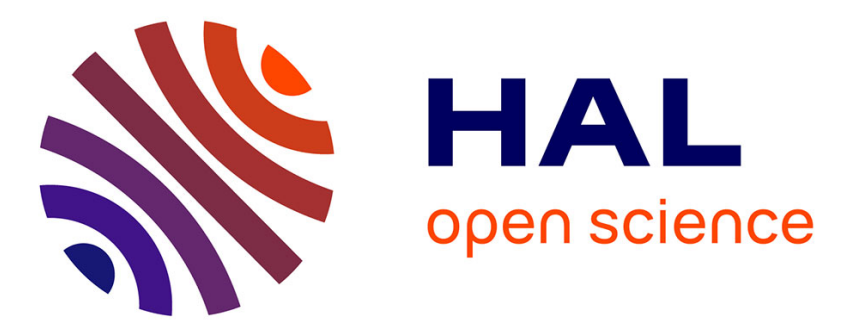

\title{
Les modalités de changement dans le système éducatif français
}

Georges Solaux

\section{To cite this version:}

Georges Solaux. Les modalités de changement dans le système éducatif français. Politiques et Management Public (Onzième colloque international, Nice, 4-5 octobre 2001), Oct 2001, Nice, France. pp.81-96. halshs-00004862

\section{HAL Id: halshs-00004862 \\ https://shs.hal.science/halshs-00004862}

Submitted on 10 Jun 2021

HAL is a multi-disciplinary open access archive for the deposit and dissemination of scientific research documents, whether they are published or not. The documents may come from teaching and research institutions in France or abroad, or from public or private research centers.
L'archive ouverte pluridisciplinaire HAL, est destinée au dépôt et à la diffusion de documents scientifiques de niveau recherche, publiés ou non, émanant des établissements d'enseignement et de recherche français ou étrangers, des laboratoires publics ou privés.

\section{(ㅇ)(1) $\$$}

Distributed under a Creative Commons Attribution - NonCommercial - NoDerivatives| 4.0 


\title{
Les modalités de changement dans le système éducatif français
}

\author{
In: Politiques et management public, vol. $20 \mathrm{n}^{\circ}$ 2, 2002. Reconfigurer l'action publique : big bang ou réforme? \\ Actes du onzième colloque international - Nice, jeudi 4 et vendredi 5 octobre 2001 - Tome 2. pp. 81-96.
}

\begin{abstract}
Résumé
Dans l'Education nationale, tout se passe comme si les décisions politiques prises étaient naturelles, comme si la solution retenue s'imposait, comme si la rationalité développée par le décideur concentrait l'ensemble des vertus traditionnellement attachées au concept même de rationalité. Mais de quelle rationalité s'agit-il en réalité ? Les études effectuées sur les systèmes éducatifs français et francophones semblent montrer que les déterminants internes aux systèmes l'emportent sur les déterminants externes, comme si la logique propre et spécifique du système éducatif constituait le moteur nécessaire et quasi suffisant de toute évolution. Les différents protagonistes développent des arguments essentiellement fondés sur leur propre idéologie et s'organisent pour structurer des décisions aux niveaux national, régional et local qui leur apportent des avantages. Le concept de causalité unique tend ainsi à s'effacer pour laisser place à la notion d'interactionnisme entre les acteurs porteurs d'intérêts divergents ou contradictoires. Le social pénètre donc l'école par les conceptions et idéologies que véhiculent les acteurs intéressés à, et parson fonctionnement. Un ministère apparaît ainsi comme un lieu de rencontre d'acteurs opposés, comme un lieu de gestion des conflits. Les arbitrages rendus par les ministres s'apparentent de ce fait souvent à des exercices de mesure des rapports de force sociaux. L'analyse des relations qui s'installent entre les niveaux de réalité macro et mésoscopiques montre que si le niveau central peut imposer un certain nombre de choix aux acteurs locaux et régionaux, en retour, les solutions proposées par ces derniers font évoluer les projets de réglementation en cours de rédaction. Le système fonctionne en autonomie quasi relative. Les acteurs semblent s'en satisfaire. Doit-on pour autant continuer à se contenter de la place laissée à l'idéologie?
\end{abstract}

Citer ce document / Cite this document :

Solaux Georges. Les modalités de changement dans le système éducatif français. In: Politiques et management public, vol. 20 $\mathrm{n}^{\circ} 2$, 2002. Reconfigurer l'action publique : big bang ou réforme ? Actes du onzième colloque international - Nice, jeudi 4 et vendredi 5 octobre 2001 - Tome 2. pp. 81-96.

doi : $10.3406 /$ pomap.2002.2718

http://www.persee.fr/web/revues/home/prescript/article/pomap_0758-1726_2002_num_20_2_2718 


\title{
LES MODALITES DE CHANGEMENT DANS LE SYSTEME EDUCATIF FRANÇAIS
}

\author{
Georges SOLAUX*
}

Résumé

Dans l'Education nationale, tout se passe comme si les décisions politiques prises étaient naturelles, comme si la solution retenue s'imposait, comme si la rationalité développée par le décideur concentrait l'ensemble des vertus traditionnellement attachées au concept même de rationalité. Mais de quelle rationalité s'agit-il en réalité ? Les études effectuées sur les systèmes éducatifs français et francophones semblent montrer que les déterminants intemes aux systèmes l'emportent sur les déterminants extemes, comme si la logique propre et spécifique du système éducatif constituait le moteur nécessaire et quasi suffisant de toute évolution. Les différents protagonistes développent des arguments essentiellement fondés sur leur propre idéologie et s'organisent pour structurer des décisions aux niveaux national, régional et local qui leur apportent des avantages. Le concept de causalité unique tend ainsi à s'effacer pour laisser place à la notion d'interactionnisme entre les acteurs porteurs d'intérêts divergents ou contradictoires. Le social pénètre donc l'école par les conceptions et idéologies que véhiculent les acteurs intéressés à, et par son fonctionnement. Un ministère apparaît ainsi comme un lieu de rencontre d'acteurs opposés, comme un lieu de gestion des conflits. Les arbitrages rendus par les ministres s'apparentent de ce fait souvent à des exercices de mesure des rapports de force sociaux. L'analyse des relations qui s'installent entre les niveaux de réalité macro et mésoscopiques montre que si le niveau central peut imposer un certain nombre de choix aux acteurs locaux et régionaux, en retour, les solutions proposées par ces demiers font évoluer les projets de réglementation en cours de rédaction. Le système fonctionne en autonomie quasi relative. Les acteurs semblent s'en satisfaire. Doit-on pour autant continuer à se contenter de la place laissée à l'idéologie?

* IREDU/CNRS Dijon.

Revue POLITIQUES ET MANAGEMENT PUBLIC, Volume 20, $\mathrm{n}^{\circ}$ 2, juin 2002.

(C) Institut de Management Public - 2002. 
Les décisions politiques concernant le système éducatif acquièrent, du fait de l'effet d'annonce voulu par les différents ministres, un caractère quasi naturel. Tout se passe comme s'il n'était pas possible de faire autrement, comme si la solution retenue s'imposait, comme si la rationalité développée par le décideur concentrait l'ensemble des vertus traditionnellement attachées au concept même de rationalité. Mais de quelle rationalité s'agit-il en réalité ? Quels sont les fondements de la décision politique ? Comment les décisions se construisent-elles ? C'est à ces questions que nous tentons d'apporter quelques éléments de réponse issus des réformes et modes de gestion de la formation initiale et continue française des années quatre-vingt et quatre-vingt dix. La place des acteurs déterminants de la décision, leurs conceptions de l'éducation, les zones de conflit générées par leurs conceptions opposées, et les arbitrages rendus par le décideur final sont définis au moyen des outils et concepts développés par la sociologie. Après avoir défini notre objet d'étude et les contextes théoriques et méthodologiques de son analyse, quelques catégories de conflits générés par les réformes du système éducatifs seront inventoriées et la formation des ingénieurs servira d'exemple à la démonstration du modèle explicatif proposé.

\section{L'école comme champ social complexe}

Notre observation est centrée sur la multitude d'acteurs individuels et collectifs qui font ou qui défont les décisions relatives aux politiques d'éducation. Les études effectuées sur le second degré semblent montrer que les déterminants internes à l'éducation l'emportent sur les déterminants externes, comme si la logique propre et spécifique du système éducatif constituait le déterminant nécessaire et quasi suffisant de toute évolution. Les différents protagonistes organisés en syndicats, associations diverses ou en corps constitués de l'administration font fréquemment valoir des points de vue divergents sur les grandes questions que soulève le développement du système éducatif. La définition de l'offre de formation, les réformes du second degré et la gestion des personnels constituent des objets de recherche qui permettent d'illustrer notre propos.

\section{- La définition de l'offre de formation}

Avec un budget voté en 2000 de 361 milliards de francs, l'Education nationale représente le premier budget de l'Etat. La dépense intérieure d'éducation qui prend en compte, outre les financements publics d'Etat, les financements des collectivités locales et des opérateurs privés, s'élève quant à elle à 625 milliards de francs. L'école, en tant qu'elle représente un objet d'allocations de ressources très importantes, n'échappe pas aux stratégies d'acteurs intéressés par les avantages financiers qu'elle est susceptible de procurer. Ces avantages se mesurent en nombre de postes d'enseignants, en budgets de fonctionnement ou d'investissement des établissement, en bourses d'études... Les enseignants, les administrateurs, les parents d'élèves, les élèves, les collectivités locales constituent autant d'acteurs intéressés par cette manne financière. Chacun veut obtenir la part qu'il estime devoir lui revenir. Ces stratégies sont partiellement guidées par la recherche de bénéfices publics certes, mais aussi privés. 
Les ètudes conduites sur les décisions politiques relatives à la carte scolaire et à la construction ou à l'équipement des établissements d'enseignement secondaire montrent que si la construction d'un collège constitue une rèponse à un besoin externe d'intérêt général (la progression démographique de l'espace considéré au regard de l'offre de formation existante), cette construction constitue aussi un ensemble de moyens que les acteurs sociaux revendiquent dans leur propre intérêt. Si l'intérêt public et l'intérêt général trouvent leur compte dans cet équipement collectif (réponse à la demande), certains intérêts particuliers s'y retrouvent aussi : les collectivités locales, les aménageurs, les constructeurs et les commerciaux voient dans cet équipement collectif un faire valoir pour leur activité : les politiques locaux le présentent comme le résultat de leur action, le parc immobilier proche sera d'autant plus valorisé qu'existent des équipements collectifs de proximité...

Les ressources publiques étant rares dans la conjoncture actuelle et à venir, les stratégies politiques attachées à l'obtention d'une part de l'offre globale de formation ne peuvent que se développer. Les différents bénéficiaires d'offre d'ores et déjà installée se mobilisent pour défendre le bien collectif qui leur est affecté, le justifier, et si possible le faire prospérer (voir sur ce plan les mobilisations sociales destinées à défendre l'école en milieu rural). Les électeurs et élus qui ne bénéficient pas d'offre de formation se mobilisent quant à eux pour obtenir une part de ce que l'on peut appeler le marché de la formation. La définition de l'offre de formation, inscrite dans les politiques publiques, n'échappe pas ainsi aux "stratégies plurielles d'acteurs sociaux euxmêmes pluriels... le concept de stratégie ...englobe l'analyse que chaque acteur, individuel ou collectif, fait des questions en débat, des points critiques, des solutions possibles, des décisions souhaitables, et de celles voisines ou contrastées, qu'il prête aux autres acteurs ${ }^{1 "}$.

\section{- Les réformes du système éducatif}

Les analyses réalisées sur la création du baccalauréat professionnel ${ }^{2}$ ou sur la réforme des lycées ${ }^{3}$ montrent que les tentatives de réforme enferment le décideur dans une contradiction qui s'exprime lorsque la recherche d'une réponse adaptée à l'expression d'un besoin collectif rencontre, d'une part, la défense des garanties statutaires enseignantes et de la fonction publique en général, et, d'autre part, les intérêts particuliers des agents concernès par les décisions retenues. Les intérêts privés s'opposent généralement aux intérêts publics, chacun s'efforçant d'obtenir dans le nouveau système des avantages supérieurs à ceux qu'il détenait dans le système antérieur. C'est dans la gestion des contradictions qui naissent de la confrontation entre le collectif national (l'état et les besoins nationaux), le collectif catégoriel (le monde enseignant ou de fonctionnaires et les représentants syndicaux), et les individus (l'enseignant objet de redéploiement et obligè de perdre une identité professionnelle pour retrouver son identité initiale; mais aussi l'élève, ses

\footnotetext{
1 Prost A. (1992), Education, société et politiques, Paris, Seuil.

2 Solaux G. (1995), Les décisions de politique éducative, le cas du baccalauréat professionnel. In Charlot, B.

Beillerot, J.. Dirs, La construction des politiques d'éducation et de formation, Paris, PUF, p. 111-128.

3 Solaux G. (1995), Le baccalauréat, Paris, La Documentation française. CNDP
} 
parents, les élus locaux...) que se trouve le politique. C'est à ce niveau que le décideur doit, en fonction des rapports de force existants, construire une réponse socialement acceptable (qui ne provoque pas des mouvements sociaux d'hostilité tels que l'Etat puisse être mis en situation de danger), politiquement réalisable (projet qui soit porté par une majorité des élus nationaux et locaux) et économiquement efficace (qui s'avère sur le plan de la relation coût - efficacité plus opérationnelle que le dispositif précédent). Le référentiel de la politique sectorielle trouve son sens dans cette articulation entre intérêts publics et intérêts privés. C'est à l'articulation de cet ensemble complexe de rapports de force que sera décidée l'inscription de la réforme à l'agenda politique.

\section{- La gestion des personnels}

Un dernier exemple issu de travaux centrés sur la gestion des personnels enseignants 1 , la gestion de leur recrutement, de leur formation et de leurs carrières, montre que ce type de politique résulte d'une équilibration progressive faisant intervenir une pluralitè d'acteurs. Ces derniers agissent soit en vue de faire progresser les avantages qui leur sont accordes, soit en vue de maintenir des avantages acquis. Les décisions de gestion des personnels enseignants sont généralement accompagnées de conflits, car les décisions prises en leur faveur ou en leur défaveur ne manquent jamais de provoquer des heurts entre groupes d'intérêts publics ou privés constitués. L'école et la gestion de ses personnels peuvent ainsi être replacées dans un contexte très général de champ social au sein duquel s'organisent des relations de pouvoir et de domination entre ministères, entre services de gestion, entre les échelons déconcentrés, entre les échelons déconcentrés et les échelons décentralisés...

A cet égard le champ social est défini par Pierre Bourdieu comme étant constitué "d'espaces structurés de positions (ou de postes) dont les propriétés dépendent de leur position et qui peuvent être analysées indépendamment des caractéristiques de leurs occupants". Le champ social est un espace où s'organisent des relations de pouvoir et de domination, structuré par un système d'enjeux, d'échanges de flux en son sein. Le concept de causalité unique tend ainsi à s'effacer pour laisser place à la notion d'interactionnisme entre les acteurs porteurs d'intérêts divergents ou contradictoires.

La recherche d'un modèle explicatif de la décision de politique éducative

Les observations précédentes semblent indiquer que l'explication de la politique publique d'éducation et de formation est peut-être inscrite dans les grands courants explicatifs de l'évolution du social dans la mesure où une

1 Solaux G. (1997), Les politiques de gestion des personnels enseignants en Afrique subsaharienne francophone. Dijon, IREDU.

Pour une expertise en management des personnels enseignants / coord. par Georges Solaux, Bruno Suchaut, Paul Dogoh-Bibi... et al. (2001), Dijon, CRDP de Bourgogne (Documents, actes et rapports pour l'éducation). 
formation peut être appréhendée comme une réalité objective (ensemble de lois et règlements, d'établissements...) existant indépendamment des acteurs, et comme une réalité subjective agie et intériorisée par les acteurs chargés de la mise en place des formations et par les bénéficiaires de la formation. Nous distinguons trois niveaux de réalité :

- le niveau macroscopique situé au niveau national et international qui rassemble les conditions économiques, sociales et politiques de l'adoption des lois et règlements destinés à réguler formellement le système. On étudie par exemple les conditions de la création du baccalauréat professionnel ou les déterminants de la décision politique nationale relative à la création des Nouvelles Formations d'Ingénieurs en 1990. Ce niveau est considéré comme le cadre structurant des formations mises en place ;

- le niveau mésoscopique qui rassemble les institutions et groupes chargés d'expérimenter puis de mettre en œuvre la politique définie au niveau local : on étudie ici les politiques académiques et régionales ou conditions de la création d'un des supports de formation des ingènieurs, les ITII (Instituts Techniques d'Ingénieurs de l'Industrie) ;

- le niveau microscopique qui concerne les unités d'enseignement (écoles, collèges, lycées et universités) ou les individus.

Au-delà de l'étude de chacun de ces niveaux de réalitè nous proposons de les articuler, de les analyser ensemble afin d'identifier ce qui dans l'un détermine le fonctionnement de l'autre. L'objectif est d'articuler ces trois niveaux d'observation qui peuvent tout à le fois être considérés comme indépendants et comme entretenant des liaisons établies par les actions en retour que chacun des niveaux peut exercer sur l'autre. La décision de politique éducative est envisagée dans une perspective théorique constructiviste qui tente de montrer comment les acteurs participent à la construction des déterminants de leur action, comment le niveau macroscopique constitue à la fois la condition et le produit de l'action des autres niveaux. Le système de formation est considéré comme évoluant en autonomie relative dans une espèce de codetermination du tout et des parties: il s'agit d'identifier comment il se construit, comment ce qui est considéré comme étant une structure résulte d'une structuration sociale qui contribue en retour à structurer le social. On tente donc de montrer que si la décision constitue une réponse de la structure de formation (changement dans l'organisation de l'école) à un besoin externe (le monde économique), elle constitue aussi un ensemble de moyens que les acteurs sociaux définissent dans leur propre intérêt. La genèse des décisions est réalisée en identifiant la place qui revient aux acteurs et au contexte économique du niveau macroscopique, et aux relations que les acteurs et contextes macroscopiques entretiennent avec les acteurs et contexte mésoscopiques et microscopiques.

Les méthodes de travail retenues
Les faits étudiés sont constitués par les pratiques de décision, et les pratiques de préparation des décisions, pratiques au sein desquelles on trouve la concertation préalable à la décision. Les pratiques de concertation sont l'occasion de développements de discours et d'actions dans, et sur l'école, 
considérés comme la matière des faits empiriques traités. Le terme du travail de recherche est atteint lorsque le modèle partiel mais construit et objectif du processus de décision est identifié. Pour y parvenir, il est possible de pratiquer par l'interview des acteurs essentiels de l'époque considérée qui fournissent leur témoignage sur la manière dont les évènements se sont déroulés, en faisant appel à leur souvenir. Cette méthode qui présente l'avantage de rendre vivante la construction opérée s'accompagne cependant du risque majeur de ne constituer qu'une somme de points de vue, d'ailleurs sélective, puisque appuyée sur le souvenir. A cette méthode nous préférons utiliser les repères sur lesquels s'appuient les historiens, c'est à dire essentiellement les documents produits à l'époque sur le sujet. Ces documents constituent des sources d'informations vérifiables et qui tombent par la recherche dans le domaine public. Elles sont donc accessibles à tous.

A cette fin nous sollicitons l'accès aux dossiers des directions ministérielles ou des collectivités territoriales. A ces dossiers essentiels, des documents d'origines diverses sont ajoutés. II s'agit en particulier des rapports produits en vue d'instruire les problèmes analysés. Ces documents permettent, comme les articles de presse de l'époque par exemple, de situer les référentiels global et sectoriel. D'une manière générale les documents suivants sont analysés:

- textes officiels réglementaires sur l'organisation de la formation des ingénieurs parus au Journal Officiel $(\mathrm{JO})$ de la République Française ou au Bulletin Officiel de l'Education Nationale (BOEN), articles de presse, publications syndicales, comptes rendus d'audiences ou de commissions au ministère de l'Education nationale, rapports officiels, sténotypie officielle des débats parlementaires (voir le Journal Officiel des débats parlementaires) et des discussions se déroulant dans les instances consultatives du ministère de l'Education nationale (Conseil National de l'Enseignement Supérieur et de la Recherche), courriers relevés dans les dossiers d'archives.

Ces faits, qui constituent l'univers empirique de la recherche, sont alors rapportés aux modèles théoriques exposés plus haut en vue de construire une intelligence de la décision de politique éducative.

Analyse des conflits générés lors de la conception des politiques éducatives
Les conflits générés par la préparation des décisions de politique éducative, que celles-ci soient prises au niveau national, régional ou local, relèvent pour la plupart de positions ou prises de position idéologiques entendues au sens le plus traditionnel. Aucun des acteurs en présence ne fournit en effet d'études objectives externes du système pour justifier ses conceptions et propositions d'organisation. Au lieu de cela à quoi assiste-t-on ? L'idéologie tend à l'emporter sur les faits, et semble fonder les décisions politiques. Louis Althusser propose que "lidéologie comme système de représentation se distingue de la science en ce que la fonction pratico-sociale l'emporte en elle sur la fonction théorique (ou fonction de connaissance)"1. Raymond Aron pense que "lidéologie est un systeme global d'interprétation historico-politique"2. Ces deux auteurs font clairement

\footnotetext{
1 Althusser L. (1965), Pour Marx, Paris, Maspero.

2 Aron R. (1966), Trois essais sur l'âge industriel, Paris, Plon.
} 
paraître l'idéologie comme système d'interprétation construit davantage en fonction des contraintes sociales que des objectifs de la connaissance scientifique, ce que $R$. Boudon propose comme relevant davantage du sens que des relations existant entre le vrai et le faux (ibid page 81 ). Nous proposons quelques exemples pour illustrer cette proposition.

\section{- La définition de la structure pédagogique et du nombre de cycles}

Ici s'opposent les "unitaires" (défenseurs d'un collège ou d'un lycée à structure identique pour tous reposant sur un nombre limité de séries) et les "pluralistes" (promoteurs d'un collège structuré sur le mode de filières ou d'un lycée organisé sur la base d'un nombre élevé de séries). L'essentiel du débat se situe dans la question qui traverse en permanence le système éducatif français depuis le développement du mouvement des compagnons de "l'université nouvelle" à l'issue de la première guerre mondiale, et la parution du rapport Langevin Wallon en 1947 : "l'objectif de l'égalisation des chances fut pris en compte par tous les gouvernements y compris les plus conservateuis. Mais le problème était de déterminer les moyens qui permettraient d'atteindre cet objectif... deux solutions antithétiques se présentent presque immédiatement à l'esprit, et elles peuvent toutes deux s'appuyer sur une argumentation solide. On peut soutenir qu'en mettant les enfants dans un moule scolaire commun aussi longtemps que possible, on se rapprochera du résultat recherché... Mais on peut utiliser une argumentation opposée : en introduisant des filières variées, on permettra à chacun de trouver chaussure à son pied ${ }^{1 "}$.

Le débat sur la gestion des élèves en difficulté et leur orientation au collège s'inscrit par exemple dans les contradictions produites par l'idéologie de ceux qui fondent la démocratisation de l'enseignement sur l'unité du système et l'idéologie de ceux qui fondent l'adaptation des èlèves au système scolaire sur la différenciation des parcours. Car d'idéologie il s'agit en effet. Quelles sont les études sérieuses, factuelles et externes au système qui permettent de déterminer les effets des différents groupements d'élèves sur l'efficacité du "traitement" des élèves en difficulté ? Quelles sont les études qui mesurent aussi les conséquences des groupements sur l'équité dans le système? Les réponses apportées à ces questions fondamentales relèvent souvent de l'intuition. Les finalités de l'orientation au collège oscillent par conséquent entre les limites accordées à la différenciation des parcours, à la plus ou moins grande rigidité de ces derniers... L'orientation au collège et en particulier celle des élèves en difficulté relève en fait de la définition que le corps social se donne, au gré de ses humeurs, de l'école moyenne : unitaire ou structurellement différencièe?

- Le nombre d'options et d'options facultatives, les identités culturelles.

La liberté dans le choix des contenus (notamment les langues vivantes et les langues anciennes) d'enseignement représente un autre axe de mobilisation

1 Boudon R. (1986), L'idéologie, Paris, Fayard. p. 154. 
des acteurs. II en est ainsi des options relatives aux langues anciennes et aux langues vivantes 3 (LV 3). Lorsqu'en 1991 Lionel Jospin, ministre de l'Education nationale, propose de limiter le nombre d'options, on assiste à la mobilisation des associations de spécialistes concernées, la Coordination Nationale des Associations Régionales des Enseignants des Langues Anciennes (CNARELA) pour le latin et le grec aricien, et la Société des Langues Néo-Latines (SLNL) pour le portugais et litalien. Les arguments développés, pour l'essentiel, par les défenseurs des langues anciennes sont présents dans une instruction du ministère de l'Education nationale du 9 août 1967: "notre langue maternelle, comme l'ensemble des langues romanes, est directement issue du latin, et c'est une vérité d'évidence que, pour la bien connaître, il est nécessaire de remonter à ses sources: l'apprentissage du latin est la meilleure façon d'accéder à la comprèhension du français", et plus loin, "aussi bien le latin n'est-il pas seulement un élément essentiel de la formation littéraire, linguistique et philosophique, définie dans la récente réforme de l'enseignement : il a naturellement sa place dans les sections scientifiques comme dans les sections littéraires conduisant au baccalauréat..."

Jacqueline de Romilly prend la tête de l'opposition au projet Jospin et crée une association en février 1992 "Sauvegarde des Enseignements Littéraires" (SEL). Mélangeant habilement la défense des "humanités classiques" avec "une régression inquiétante dans l'enseignement du français lui même", cette association ètend le problème de quelques latinistes et hellénistes à l'ensemble des lycèens. Les membres de l'association sont particulièrement actifs et comptent dans leurs rangs des signatures jugées comme illustres : Pierre Grimal, Jean Bernard, Hèlène Carrère d'Encausse, Michel Droit, André Frossard, Bertrand Poirot-Delpech, Jean-François Revel, Léopold SedarSenghor... Ces personnalités s'expriment sur le sujet dans la presse en faisant valoir des points de vue proches de ceux exprimés par l'instruction du 9 août 1967 mais supportés par un vocabulaire plus dramatique. Le journal "le quotidien de Paris" se fait volontiers leur interprète (voir la parution du 12 mars 1992): " voici toutes les raisons pour lesquelles il faut apprendre la latin et pour lesquelles je suis contre cette réforme dangereuse : le français vient du latin et pour connaître le français il faut connaître le latin, le latin a des formes de pensée très différentes des autres langues... pour apprendre le latin il faut faire des efforts qui musclent l'esprit... la plupart des grands scientifiques d'autrefois étaient de grands latinistes, on prétend que notre époque est scientifique. Or le latin est une matière scientifique, elle développe l'esprit scientifique. C'est criminel de tuer le latin..." (Paul Guth)... " je suis plus que tout partisan du latin et du grec. On ne peut pas apprendre le français, être fidèle à la langue française par laquelle nous pensons, nous aimons, sans être fidèle au latin... Les enfants de France ne connaissent pas l'orthographe, même les candidats à l'agrégation ne savent pas écrire le français. Cette réforme est très grave et lamentable, elle affaiblit toute la francophonie, voire plus encore..." (Jean Guitton).

Le mouvement de protestation s'arrête lorsque Jack Lang, successeur de Jospin, propose de réinstaller les langues anciennes comme option facultative en classe de seconde et de permettre aux élèves de choisir deux options

1 BOEN du 31 août 1967. 
facultatives au baccalauréat. La part d'idéologie qui sous-tend les décisions prises à ce niveau sont illustrées par le fait que François Bayrou acceptera trois options facultatives au baccalauréat, Claude Allègre revient en arrière en 1997 et limite à une seule option facultative tandis que Jack Lang en 2000 repasse à deux options. Où sont donc les justifications objectives de telles variations dans les prises de décision de cinq ministères successifs en dix ans?

Les identités culturelles font l'objet de luttes ardentes de la part des défenseurs de certaines disciplines et des diverses associations de spécialistes enseignantes lors de la définition des horaires d'enseignement. La défense des identités culturelles est réalisée par ceux qui, ayant bénéficié de l'école, souhaitent retrouver dans celle-ci les mêmes caractéristiques que celles qu'ils y ont rencontrées lorsqu'ils étaient élèves. Ne pas retrouver dans l'école actuelle la même culture que celle dont on a bénéficiè dans l'école de sa jeunesse, qui fut et qui est constitutive de ce que l'on est aujourd'hui, qui est constitutive de l'identité propre, amène à se sentir exclu de la culture dominante actuelle et donc à se sentir nié dans son existence. II y va de la survie sociale, de la continuité de la reconnaissance de soi. Ceci explique les violentes réactions de la part des défenseurs des Certificats d'Aptitudes Professionnelles (CAP) en 1985 et des défenseurs du latin en 1992 par exemple.

\section{- La professionnalité enseignante}

Le sens à donner à l'enseignement secondaire et à la professionnalité enseignante est le troisième point d'ancrage des oppositions et conflits : l'enseignement secondaire consiste-t-il fondamentalement à transmettre des connaissances dans le cadre strict des disciplines d'enseignement ou bien peut-il y avoir place pour des activités pédagogiques destinées à doter les élèves de "technologies intellectuelles" 1 " (opposition entre les défenseurs du "tout disciplinaire" et les "pédagogues") ? Les débats les plus vifs sur ce sujet se produisent lors des discussions relatives à la définition des méthodes pédagogiques et des épreuves du baccalauréat. Ces deux sujets sont éminemment représentatifs de la professionnalité enseignante.

Le premier voit s'opposer les défenseurs d'un enseignant, traditionnellement seul dans sa classe, et compris fondamentalement comme un agent de transmission des connaissances, aux promoteurs d'un enseignant travaillant en équipe et davantage tourné vers la transmission de méthodes de travail ou de technologies intellectuelles. Au niveau du baccalauréat ce sont les défenseurs de l'examen sous forme de contrôle ponctuel terminal, avec des épreuves écrites anonymes, qui se sont opposés aux promoteurs du contrôle en cours de formation. L'identité enseignante dominante dans les lycèes, celle qui est défendue par les organisations professionnelles nationales majoritaires aux élections professionnelles, est donc constituee d'une image fortement ancrée dans un champ disciplinaire avec refus de tout contrôle social effectué par les pairs (refus du travail en équipe et du contrôle en cours de formation).

1 Bourdieu P. (1985), Rapport au Président de la république, (Collège de France) 


\section{- Horaires et emplois du temps des élèves}

La définition de l'espace culturel des lycées par le nombre de disciplines d'enseignement et les horaires hebdomadaires qui sont alloués à chacune d'elles représente un enjeu supplémentaire de prise de pouvoir dans la décision. Il faut par exemple savoir que les collèges publics et privés sont composés de 135000 classes. Imaginons que le ministre propose la diminution d'une heure de cours en mathématiques sur les quatre niveaux qui vont de la sixième à la troisième pour alléger les emplois du temps: l'enseignement de mathématiques diminuerait en conséquence de 135000 heures par semaine (une heure par semaine dans l'emploi du temps hebdomadaire de chaque classe). Chaque professeur de collège assure environ 20 heures de cours hebdomadaires. Une diminution de 135000 heures de cours consisterait en réalitè à supprimer 6750 postes. Peut-on imaginer que les syndicats, les associations de spécialistes concernées demeurent indifférents à cette proposition ? La sélection des savoirs scolaires, leurs modes de diffusion et de validation auprès des élèves, ne s'opèrent pas dans des espaces sociaux neutres. Ceux-ci sont structurés par la présence d'un Etat, de syndicats, d'associations et de mouvements divers au sein desquels des acteurs dominants exercent leur pouvoir d'imposition tant à l'Etat qu'aux autres acteurs. La définition des disciplines d'enseignement et des horaires hebdomadaires est également l'occasion d'identifier, au sein même de l'Administration Centrale de l'Education Nationale, des catégories d'acteurs administratifs (inspections générales par exemple) qui, profitant des incertitudes provoquées par l'attente des prises de décision, tentent de faire valoir leur point de vue afin de tirer quelque bénéfice supplémentaire de l'organisation à venir.

\section{- La réforme du baccalauréat}

La définition de l'examen terminal du baccalaureat fait l'objet de mobilisations sociales très importantes. Parce que le baccalauréat est tout à la fois la reconnaissance de l'accès à la culture et la clef d'accès à l'université c'est à dire à LA culture, il occupe une place particulière dans les représentations. II doit, au sens normatif du terme, présenter des caractéristiques propres qui le rendent indiscutable, juste, et équitable. II doit apparaître "comme le modèle de toute certification scolaire. Du même coup toute tentative pour le remettre radicalement en cause paraît d'avance vouée à l'échec. Même les formules, pourtant modestes, d'adaptation ou de correction du baccalauréat qui ont été proposées depuis un quart de siècle n'ont pas pu grand chose contre la logique propre de l'examen 1 ".

La place du baccalauréat semble donc centrale dans le dispositif de l'enseignement de second degré français, il doit présenter les caractéristiques attendues de ce que doit être un véritable examen et il représente une symbolique de ce que sont les comportements des enseignants. Modifier l'organisation du baccalauréat c'est donc modifier les critères de reconnaissance de la culture et les éléments constitutifs de cette dernière. C'est en réalité dire aux

1 Prost A. (1983), Les lycées et leurs études au seuil du XXime siècle, Paris, CNDP, p. 133. 
détenteurs de la culture dominante, que sont les enseignants, que les principes autour desquels ils ont construit leur identité culturelle doivent être revus. Les enjeux sont ainsi considérables et l'on peut s'attendre à des résistances qui dépassent celles que nous avons analysées jusqu'ici. C'est sans doute pour cette raison que les ministres successifs hésitent dans la mise en oeuvre d'une réforme de ce type.

Le social pénètre donc l'école par les conceptions et idéologies que véhiculent les acteurs intéressés à, et par son fonctionnement. Un ministère apparaît ainsi comme un lieu de rencontre d'acteurs opposés, comme un lieu de gestion des conflits. Les arbitrages rendus par les ministres s'apparentent de ce fait souvent à des exercices de mesure des rapports de force sociaux.

Les conflits provenant de la mise en œuvre des réformes
Le ministère de l'Education nationale fait gènéralement précéder la mise en place des réformes par ce qu'il appelle une expérimentation. II en a été ainsi de la création du baccalauréat professionnel, de la réforme des lycées et notamment de la mise en place des "modules" en classe de seconde, et de la réforme des collèges initiée par François Bayrou. II s'agit en réalité de proposer à un échantillon restreint d'établissements volontaires de mettre en place la réforme en question. Le ministère de l'Education ne peut se passer du terrain pour mettre à l'épreuve sa politique publique innovante, pour donner de la consistance à ce qui ne resterait qu'un objet de discours politique sans la réalisation permise par l'expérimentation. Le partenaire local, en expérimentant un dispositif nouveau conçu par le niveau national, participe à la construction de la modernité et en tire un bénéfice d'image médiatique important. Cependant, ce souci de tester le réel face au changement souffre de problème méthodologiques importants. C'est par exemple l'année qui précède la généralisation que la dite expérimentation est conduite ce qui amène les ministres à décider en janvier la généralisation pour la rentrée de septembre alors que les conséquences de la mise en œuvre des nouvelles modalités de fonctionnement ne sont pas connues. Par ailleurs il apparaît clairement que ce type d'actions recense davantage, lors de l'évaluation de l'expérimentation, les opinions des acteurs enseignants et administratifs sur les difficultés qu'ils ont eux-mêmes rencontrées, que les effets induits sur les élèves (en terme d'effets positifs ou négatifs).

Cette manière de procéder, sans réglementation nationale préexistante et sans définition précise du cadre juridique du dispositif associant les partenaires, est peut-être la plus efficace en matière de gestion des politiques publiques innovantes, car d'une certaine manière elle contraint les partenaires à s'entendre sur les modalités juridiques à mettre en oeuvre pour faire fonctionner le dispositif. Elle présente cependant un risque considérable pour les bénéficiaires que sont les élèves. II est en effet tout à fait possible d'imaginer qu'une réforme soit jugée porteuse d'effets positifs, alors que ces derniers ne le sont que pour les enseignants et que le contraire se produise pour les èlèves. En réalité, il nous semble que les expérimentations ont pour objectif essentiel de vérifier la faisabilité des actions réformées beaucoup plus que leur efficacité. Les relations entre les niveaux macro, méso et microscopiques s'installent lors de ces périodes d'expérimentation. Pour 
illustrer ce point nous proposons d'analyser la mise en place des Nouvelles Formations d'Ingènieurs (NFI).

A la suite du rapport Decomps sur la formation d'ingénieurs, l'année 1990 voit se répandre l'idée qu'"il faut doubler le flux des ingénieurs pour devenir compétitif", en les formant selon le modèle allemand. La simplicitè de l'idée explique sans doute qu'elle ait été largement diffusée par la presse pendant quatre années de 1989 à 1992 . L'opinion publique était acquise aux mesures proposées par le politique, d'autant que le Président de la République a alors pesé de toute sa légitimité sur ce point (en revenant sur son souhait de doubler le flux de sortie des ingénieurs en quatre années lors de son intervention publique à Evry). II y a là place pour la naissance d'une idéologie car si des études scientifiques ont été produites, la vérité qu'elles ont produite a été socialement "falsifiée" par des simplifications abusives destinées à rendre "lisible" le message comme suit : "la France ne forme pas assez d'ingénieurs, ceux qu'elle forme ne conviennent pas aux PME-PMI, il faut donc doubler le flux de formation, en formant les nouveaux venus selon un nouveau standard copié sur le modèle allemand".

La situation du contexte de création de la NFI permet de mettre en évidence trois éléments importants pour comprendre la rapidité avec laquelle la NFI verra le jour dans une ville dont l'importance démographique et industrielle ne laissait pas supposer au départ que cela fût possible: Auxerre. Le premier élément concerne la création préalable d'une Maîtrise de Sciences et Techniques (MST) à l'université de Bourgogne, dont les formations professionnelles sont organisées dès la création en 1988 à la Maison de l'entreprise d'Auxerre avec l'appui de la municipalité. La création de la NFI s'inscrit par conséquent dès le départ dans un environnement dynamique fondé sur une réalisation commune préalable entre les partenaires principalement intéressés (municipalité, organisation professionnelle patronale, université...). Le second élément se situe au niveau de la stature, alors nationale, du Maire de la ville Jean Pierre Soisson qui, outre ses fonctions de premier magistrat de la cité, occupait en 1986 le poste de vice-président du Conseil régional de Bourgogne chargé de la formation professionnelle et de l'apprentissage et qui en 1988 se voit attribuer le portefeuille de ministre du Travail et de la Formation professionnelle. L'Université de Bourgogne, enfin, joue le jeu de l'ouverture à deux niveaux. D'une part elle accepte d'envisager l'implantation d'un second cycle universitaire hors du chef lieu, alors que traditionnellement ce sont les premiers cycles qui sont concernés par ce qu'il est convenu d'appeler depuis la délocalisation. D'autre part elle participe activement à une collaboration avec les milieux professionnels, allant jusqu'à installer les enseignements professionnels de la MST dans des locaux appartenant à des organisations professionnelles. L'université en 1986-1987 s'est résolument tournèe vers le développement régional tout en maintenant par ses exigences et contrôles pédagogiques la valeur des diplômes nationaux qu'elle délivre. Un juste équilibre semble à l'époque avoir été trouvé entre des exigences académiques et donc universelles par principe et les besoins locaux ancrés dans le temps présent.

La création de la NFI et les enjeux qui en sont nés nous semblent reposer pour l'essentiel sur la définition des statuts des Instituts Techniques d'Ingénieurs de l'Industrie (ITII). Ces statuts n'existaient pas avant la 
publication du rapport Decomps, et ce n'est qu'à l'issue de la publication de ce dernier que l'Union des Industries Métallurgiques et Minières (UIMM, branche puissante du Conseil National du Patronat Français de l'époque : CNPF) a proposé leur création souhaitant ainsi disposer de structures de formation clairement identifiées comme relevant des organisations professionnelles patronales. Nous avons observé que des relations conflictuelles s'étaient installées entre la Direction des Enseignements Supérieurs (DESUP au ministère de l'Education nationale) gardienne de l'orthodoxie en matière de formations supérieures, et I'UIMM soucieuse de faire évoluer le cadre de la formation des ingénieurs vers davantage de souplesse, vers davantage de libéralisme. C'est dans le cadre de ces conflits que les relations macro et mésoscopiques s'installent.

Alors que les politiques et l'administration de l'Education nationale sont mobilisés par la mise en place des nouvelles formations, I'UIMM se mobilise quant à elle pour tirer bénéfice des décisions politiques relatives aux NFI en tentant de fair z reconnaître les associations ITII comme écoles d'ingénieurs (par l'apprentissage et la formation continue). La reconnaissance des ITII comme établissements d'enseignement disposant du pouvoir de délivrer un diplôme d'ingénieur situerait l'UIMM (et donc le CNPF) comme acteur dans le champ des certifications supérieures en brisant le monopole de la collation des grades de l'état. Un véritable "bras de fer" va s'engager entre l'UIMM et la Commission du Titre d'Ingénieur (CTI) (favorable aux prises de position de I'UIMM) d'une part, et la DESUP d'autre part, sur la dénomination du titre d'ingénieur formé selon les principes "NFI". Car si la dénomination du titre d'ingénieur formé sur le support des ITII fait apparaître ces derniers comme délivrant le diplôme, les ITII deviennent établissements d'enseignement supérieur privès, et par la même occasion I'UIMM devient support d'établissements d'enseignement supérieur.

Bien que le projet de statut de l'ITII d'Auxerre soit disponible dès septembre 1990, il ne sera adopté par l'assemblée générale de l'ITIl que le 5 mars 1991. Entre ces deux dates les diverses organisations nationales et locales sont entrées en contact en vue de réaliser des statuts qui fussent compatibles avec les intérêts de l'Etat gestionnaire de l'enseignement supérieur et ceux des organisations professionnelles soucieuses de voir leurs compétences en matière de formation de cadres supérieurs reconnue par la voie de le formation continue, mais surtout par celle de l'apprentissage. Les statuts de I'ITII vont être discutés dans un contexte qui présente deux caractéristiques conflictuelles. D'une part, on note une volonté de prise de responsabilités par les chambres syndicales patronales et, d'autre part, on observe le souci exprimé par l'administration centrale de l'Education nationale de sauvegarder son monopole de la délivrance des diplômes. Nous disposons, pour illustrer l'intensité des interactions entre les acteurs locaux et nationaux, de la lettre de soutien de J.P. Soisson, Ministre du Travail et de la Formation Professionnelle, au Président de l'Université de Bourgogne sur le problème de la délivrance du diplôme (lettre du 15/02/91) : "Je souhaite que l'université de Bourgogne conserve au niveau de la délivrance du titre d'ingénieur la maîtrise de l'opération que nous avons tous deux engagée à Auxerre... S'il le faut vous savez que je suis prêt à tenir "lit de justice" pour qu'un accord intervienne sur la base de notre volonté commune". Le débat entre les promoteurs de l'entreprise formatrice et le ministère de l'Education nationale qui a très 
longtemps disposé du monopole de la formation et de la définition des diplômes, trouve donc un champ d'application nouveau avec la définition des ITIl et la responsabilité de la délivrance du diplôme.

Les négociations locales sont âpres et le président de l'Université de Bourgogne tout en étant le relais de la DESUP au niveau local, contribue, par l'information qu'il fait " remonter » au ministère, à façonner les représentations des responsables administratifs et politiques du niveau national. Appuyé par les représentants syndicaux d'enseignants en Conseil d'administration de l'université de Bourgogne, le président est déterminé à conserver le contrôle régional de la reconnaissance des acquis sous forme de diplôme. Cette résistance régionale et la détermination des syndicats et responsables de l'université ont conforté les prises de position de la DESUP face à I'UIMM. Lionel Jospin clôt le débat le 28 janvier 1992 en adressant un courrier particulièrement bref au Président de I'UIMM (ref cab 446) qui ne fait que reprendre les termes du courrier adressé par son Directeur de cabinet adjoint un an et demi plus tôt: "je ne peux que confirmer les termes de la lettre du 26 novembre 1990 : "Je suis heureux que nous ayons pu nous accorder sur une dénomination du titre : "diplôme d'ingénieur de l'ITII de ... délivré par IUniversité (ou l'école) de ..." Je tiens en effet que soit clairement marquée la responsabilité propre du partenaire pédagogique sans que les principes d'un partenariat équilibré, tel que vous le concevez, ne soient entamés. Cela évite à considérer comme des écoles techniques privées, au sens du code de l'enseignement technique, les associations que vous souhaitez créer, structures support de ce partenariat et de gestion".

L'étude de l'implantation de la politique NFI à Auxerre ne peut donc se limiter à l'analyse du processus de mise en oeuvre, au niveau local, de la politique qui a été décidèe au niveau national. En effet, pour qu'il y ait simple mise en oeuvre d'une décision politique, il faudrait accepter l'idée que les acteurs locaux sont de simples exécutants, des rouages sans responsabilité qui sont limités à l'application de directives venues "d'en haut". La détermination directe de l'action auxerroise par le niveau national nous semble trop simple sur le plan sociologique, ou plus précisément trop linéaire. La notion de chaîne hiérarchique, dans le cas qui nous occupe, explose littéralement du fait de la présence d'acteurs soit relevant de champs sociaux différents soit appartenant à plusieurs champs dans le même temps. Lorsque les acteurs occupent l'espace mésoscopique auxerrois, chacun d'entre eux entretient des relations verticales spécifiques avec sa hiérarchie située au niveau macroscopique national. L'Université de Bourgogne est en relation avec le ministère de l'Education nationale et plus particulièrement la Direction des Enseignements Supérieurs (DESUP). Les professionnels locaux sont en relation avec leurs fédérations professionnelles nationales et en particulier I'UIMM. Mais si ces acteurs sont en relation avec leur " hiérarchie " dont ils défendent les intérêts et positions, ils disposent de marges de liberté, de pouvoirs statutaires (autonomie des universités par exemple), qui leur permettent d'ètre des acteurs effectifs dans la définition de structures, des lois et règlements qui vont organiser leu: fonctionnement. La mairie d'Auxerre aurait pu, quant à elle, être relativement autonome dans ce jeu de dépendance hiérarchique si son maire Jean-Pierre Soisson n'avait èté à l'époque Ministre du travail et de la Formation Professionnelle dans le gouvernement de Michel Rocard. JeanPierre Soisson appartenant aux espaces macroscopiques et mésoscopiques 
est porteur des intérêts et contradictions des deux niveaux: l'opportunité de la création d'une formation innovante dans sa ville ne lui a pas échappé, les intèrêts macroscopiques rejoignent par sa personne les intérêts mésoscopiques.

L'analyse des relations qui se sont alors installées entre le niveau mésoscopique et le niveau macroscopique montre que si le niveau national a pu imposer un certain nombre de choix aux acteurs locaux et régionaux, en retour, les solutions proposees par ces derniers, notamment à l'occasion de la définition des statuts des ITII, ont fait évoluer les projets de réglementation en cours de rédaction. La structuration de la formation des ingénieurs par apprentissage ne résulte donc pas seulement de conflits arbitrés au niveau national par le ministère mais aussi des niveaux mésoscopiques qui tout en étant structurés dans leurs démarches par les projets de réglementation macroscopique parviennent par leurs actions en retour, à participer à la définition de la structure qui les contraint.

\section{Conclusion}

Alors que l'ambition des ministres est de mettre le système éducatif au moins partiellement au service de l'économie et du social, c'est à dire de besoins externes clairement identifiés, force est de constater que leurs décisions sont davantage fondées sur l'arbitrage entre les intérêts contradictoires véhiculés par les acteurs internes du système. Tout semble se passer comme si le système éducatif fonctionnait en autonomie relative par rapport aux contraintes économiques et sociales. La force des partenaires sociaux dominants s'exprime d'autant plus que l'Education nationale constitue le réservoir de la culture dominante du futur et un réservoir de ressources publiques et privées énorme dont chacun d'eux pense légitimement pouvoir disposer. Quatorze millions de personnes (élèves, étudiants et personnels enseignants et administratifs) vivent dans et de l'école, c'est-à-dire un quart environ de la population du pays. Ces masses démographiques, budgétaires et culturelles peuvent difficilement se gérer sur la seule base d'une appréciation externe et objective des faits comme le voudrait une démarche fondée sur la recherche d'une relation plus équilibrée entre les coûts et l'efficacité du système. Doit-on pour autant continuer à se contenter de la place laissée à l'idéologie ? 


\section{BIBLIOGRAPHIE}

(1996) "Les groupes d'intérêt ", Revue Pouvoirs n 79, Seuil, Paris.

ARDOINO J., J. BERGER, G. (1989), «D'une èvaluation en miettes à une évaluation en actes ",

ALTHUSSER L. (1965), Pour Marx, Paris, Maspero.

ARON R. (1966), Trois essais sur l'âge industriel, Paris, Plon.

BERTHELOT J.M. (1990), L'intelligence du social, Paris, PUF.

BOUDON R. (1979), La logique du social : introduction à l'analyse sociologique, Paris, Hachette.

BOUDON R. (1986), L'idéologie, Paris, Fayard.

BOURDIEU P. (1985), Rapport au Président de la république, (Collège de France).

BOURDIEU P. (1987), Le sens commun, Paris, Editions de minuit.

BRAUD P. (1994), Sociologie politique, Paris, Editions LGDJ.

CHEVALLIER J. (1994), Le service public, Paris, PUF.

Commissariat Général du Plan. (1991), Outils, pratiques, institutions pour évaluer les politiques publiques, La Documentation Française, Paris.

CROZIER M. (1977), L'acteur et le système, Seuil, Paris.

FORQUIN J.C. (1989), Ecole et culture, Bruxelles, De Boeck.

HABERMANS J. (1973), La technique et la science comme idéologie, Paris, Gallimard.

HOBSBAWN EJ. (1999), L'âge des extrêmes, histoire du court vingtième siècle, Editions complexes, Bruxelles.

LACASSE F., THOENIG JC. (1996), L'action publique, L'Harmattan, coll Logiques politiques, Paris.

LECOMTE P., DENNI B. (1990), Sociologie du politique, Presses universitaires de Grenoble.

MULLER P. (1990), Les politiques publiques, Paris, PUF.

PROST A. (1983), Les lycées et leurs études au seuil du XXf siècle. Paris, CNDP.

PROST A. (1992), Education, société et politique, Paris, Seuil.

SOLAUX G. (1990), La création du baccalauréat professionnel : faits économiques et sociaux, stratégies des acteurs, Thèse de doctorat, Dijon, Universitè de Bourgogne, décembre 1990.

SOLAUX G. (1995), Le baccalauréat, Paris, La Documentation Française, CNDP.

SOLAUX G. (1995), Les décisions de politique èducative : le cas du baccalauréat professionnel. In CHARLOT B., BEILLEROT J., Dirs, La construction des politiques d'éducation et de formation, Paris, PUF, $p$. $111-128$.

SOLAUX G. (1997), Les politiques de gestion des personnels enseignants en Afrique subsaharienne francophone, Dijon, IREDU.

Pour une expertise en management des personne/s enseignants / coord. par Georges SOLAUX, Bruno SUCHAUT, Paul DOGOH-BIBI...et al., (2001), Dijon, CRDP de Bourgogne (Documents, actes et rapports pour l'éducation).

TOURAINE A. (1984), Le retour de l'acteur, Paris, Fayard. 